Article

\title{
Effect of Bucillamine on Free-Radical-Mediated Degradation of High-Molar-Mass Hyaluronan Induced in vitro by Ascorbic Acid and $\mathrm{Cu}(\mathrm{II})$ Ions
}

Mária Baňasová ${ }^{1, *}$, Katarína Valachová ${ }^{1}$, Jozef Rychlý ${ }^{2}$, Ivica Janigová ${ }^{2}$, Katarína Csomorová ${ }^{2}$, Raniero Mendichi ${ }^{3}$, Danica Mislovičová ${ }^{4}$, Ivo Juránek ${ }^{5}$ and Ladislav Šoltés ${ }^{5}$

1 Institute of Experimental Pharmacology and Toxicology, Slovak Academy of Sciences, SK-84104 Bratislava, Slovakia; E-Mail: katarina.valachova@savba.sk

2 Polymer Institute, Slovak Academy of Sciences, SK-84541 Bratislava, Slovakia;

E-Mails: jozef.rychly@savba.sk (J.R.); ivica.janigova@savba.sk (I.J.); katarina.csomorova@savba.sk (K.C.)

3 Istituto per lo Studio delle Macromolecole, Consiglio Nazionale delle Ricerche, I-20133 Milano, Italy; E-Mail: mendichi@ismac.cnr.it

4 Institute of Chemistry, Slovak Academy of Science, SK-84538 Bratislava, Slovakia; E-Mail: danica.mislovicova@savba.sk

5 Institute of Experimental Pharmacology and Toxicology, Slovak Academy of Sciences, SK-84104 Bratislava, Slovakia; E-Mails: ivo.juranek@savba.sk (I.J.); ladislav.soltes@savba.sk (L.S.)

* Author to whom correspondence should be addressed; E-Mail: banasova.majka@gmail.com; Tel.: +421-910-928-634; Fax: +421-254-775-928.

External Editor: Christine E. Schmidt

Received: 22 July 2014; in revised form: 23 September 2014 / Accepted: 14 October 2014 / Published: 21 October 2014

\begin{abstract}
The bucillamine effect on free-radical-mediated degradation of high-molar-mass hyaluronan (HA) has been elucidated. As HA fragmentation is expected to decrease its dynamic viscosity, rotational viscometry was applied to follow the oxidative HA degradation. Non-isothermal chemiluminometry, thermogravimetry, differential scanning calorimetry, and size-exclusion chromatography (SEC) were applied to characterize resulting HA fragments. Although bucillamine completely inhibited the HA viscosity decrease caused by oxidative system, indicating HA protection from degradation, SEC analysis suggested that some other mechanisms leading to the bucillamine transformations without the decay of the viscosity may come into a play as well. Nonetheless, the link between the reduction of
\end{abstract}


chemiluminescence intensity and disappearance of the differential scanning calorimetry exotherm at $270{ }^{\circ} \mathrm{C}$ for fragmented HAs indicates a particular role of the bucillamine in preventing the decrease of HA viscosity.

Keywords: disease-modifying antirheumatic drugs; rotational viscometry; size exclusion chromatography; thermo-analytical methods

\section{Introduction}

Hyaluronic acid or its salt (Figure 1a); (HA) is a saccharidic biopolymer composed of regularly alternating units of $N$-acetyl-D-glucosamine and D-glucuronic acid linked by $\beta-(1 \rightarrow 3)$ and $\beta$ - $(1 \rightarrow 4)$ linkages. In the body of vertebrates, HA is present in extracellular matrices, mostly associated with various proteoglycans. In the associated form, HA macromolecules function as a scaffold, thus, forming more or less hard tissue(s) — cartilage, umbilical cord, and skin. In vitreous humor, HA chains fill the space between the collagen fibrils providing an elastic character to this soft tissue [1-5].

Figure 1. (a) Hyaluronan acid; (b) bucillamine; and (c) $N$-acetyl-L-cysteine.

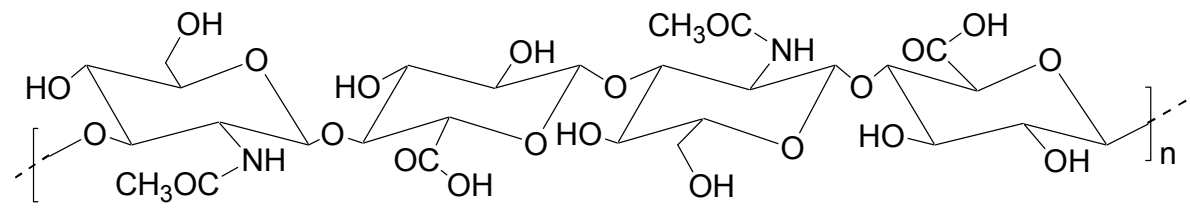

a)<smiles>CC(C)(S)C(=O)N[C@@H](CS)C(=O)O</smiles>

b)<smiles>CC(=O)N[C@@H](CS)C(=O)O</smiles>

c)

Synovial fluid (SF), of which viscoelastic properties are governed by HA of megaDalton molar mass, protects the articular cartilage from mechanical impacts [6,7]. In contrast to all the above-mentioned tissues, the viscoelasticity of SF is related to unassociated high-molar-mass HAs dissolved molecularly within this fluid [8,9].

A 70-kg human body contains approximately $15 \mathrm{~g}$ of HA [10]. Every day about one third of this amount is turned over. The two modes of HA decay have to be mentioned here: First, enzymatic "depolymerization", by which HA fragments of lower molar size are generated, and second, oxidative degradation, a process which has been intensively studied by biochemists, molecular biologists, and polymer chemists.

The half-life of HA varies from two to five minutes in blood plasma, one to two days in the skin, and up to one to three weeks in cartilage [11,12]. The turnover of HA in the above mentioned tissues is controlled enzymatically by hyaluronidases (HYALs). On the other hand, in SF of healthy humans, whose fluid is free of any HYALs, the half-life of HA, equaling about $12 \mathrm{~h}$, is suggestive of other than 
enzymatic decay. One explanation of such a fast HA turnover in SF could be a "mechanistic" pumping out of a part of SF through the lymph drainage system during the daytime moving activity of the person. Another mechanism proposed recently is a particular oxidative degradation of MDalton HAs in SF of healthy humans $[13,14]$. The latter process, however, should be dominant in arthritic diseases such as osteoarthritis and especially in rheumatoid arthritis (RA). In the SF of patients suffering from RA, a significant HA molar-mass reduction has been diagnostically proved.

To cure RA patients, several disease-modifying antirheumatic drugs (DMARDs) have been applied. The relevant question may be put forward: Are these drugs acting also as antioxidants? If so, do these drugs protect the HA from oxidative degradation?

In continuation of previous study [15] where the effect of $N$-acetyl-L-cysteine (NAC) on HA degradation due to Weisberger biogenic oxidative system (WBOS) [16,17] comprising ascorbic acid plus $\mathrm{Cu}(\mathrm{II})$ ions was examined, this study was aimed at the modifications in HA macromolecules exposed to the WBOS in the absence and presence of bucillamine $N$-(2-mercapto-2-methylpropionyl)-L-cysteine (Figure 1b). Bucillamine was supposed to be significantly more efficient antioxidant than the parent NAC. The reason for this consists in two SH groups in a one molecule while NAC has only one. Moreover, the HA fragments after the degradation due to WBOS in the presence of NAC performed quite unique chemiluminescence and DSC (differential scanning calorimetry) patterns when compared with other thiols examined [15], which was also worth of further study. It may be of interest that bucillamine and NAC have the similar structural moiety with $\mathrm{N}$-acetyl group of glucosamine units in HA macromolecules. Changes in HA regarding its molar-mass were examined in detail by using size-exclusion chromatography with multi-angle light scattering (SEC-MALS) analysis. Structural changes of HA after its fragmentation and apparent removal of any low molar mass additive by back precipitation of the solid HA were searched for using several thermo-analytical methods, such as non-isothermal chemiluminometry, differential thermogravimetry (DTG), and DSC.

\section{Experimental Section}

\subsection{Chemicals}

A high-molar-mass hyaluronan sample (sodium salt) coded here P0207-1A, purchased from Lifecore Biomedical Inc. (Chaska, MN, USA) was characterized by SEC-MALS (Table 1). Analytical purity grade $\mathrm{NaCl}$ and $\mathrm{CuCl}_{2} \cdot 2 \mathrm{H}_{2} \mathrm{O}$ were purchased from Slavus Ltd. (Bratislava, Slovakia). Bucillamine was the gift of Santen Pharmaceutical Co. (Osaka, Japan). L-ascorbic acid was the product of Merck KGaA (Darmstadt, Germany). Ethanol (96\%) of p.a. purity grade was purchased from Mikrochem, Pezinok, Slovakia. Redistilled deionized high quality grade water, with conductivity of $<0.055 \mathrm{mS} / \mathrm{cm}$, was produced using the TKA water purification system from Water Purification Systems GmbH (Niederelbert, Germany).

\subsection{Rotational Viscometry}

The dynamic viscosity of the reaction mixture $(8 \mathrm{~mL} ; 0.15 \mathrm{~mol} / \mathrm{L}$ aqueous $\mathrm{NaCl})$ containing $\mathrm{HA}$ $(20 \mathrm{mg})$, ascorbic acid $(100 \mu \mathrm{mol} / \mathrm{L})$ plus $\mathrm{Cu}(\mathrm{II})$ ions $(1 \mu \mathrm{mol} / \mathrm{L})$ either without (coded i) or with (coded ii, iii) addition of $100 \mu \mathrm{mol} / \mathrm{L}$ bucillamine was monitored by a Brookfield LVDV-II+PRO digital 
rotational viscometer (Brookfield Engineering Labs., Inc., Middleboro, MA, USA) at $25.0 \pm 0.1{ }^{\circ} \mathrm{C}$ and at a shear rate of $237.6 \mathrm{~s}^{-1}$ for $5 \mathrm{~h}$ in a reservoir-spindle couple made of Teflon ${ }^{\circledR}$. The drug was introduced into the reservoir vessel at start of the reaction (ii) or $1 \mathrm{~h}$ after the reaction onset (iii). Five hours later, the reaction mixture $(8 \mathrm{~mL})$ was poured into $20 \mathrm{~mL}$ ethanol, centrifuged, and the precipitate was dried in a desiccator. The yields of the precipitated polymers were (i) $17.8-18.4 \mathrm{mg}$, (ii) $18.7-19.4 \mathrm{mg}$, or (iii) $17.0-18.2 \mathrm{mg}$.

\subsection{Simple SEC Analyses}

Evaluation of the HA molar mass changes, running under the conditions given above at monitoring the dynamic viscosity of the reaction mixture, was performed simply with a Shimadzu (Kyoto, Japan) apparatus using a packed HEMA-BIO 1000 column (Tessek, Prague, Czech Republic) of dimensions $7.8 \mathrm{~mm} \times 250 \mathrm{~mm}$; the packing particle size was $10 \mu \mathrm{m}$. The mobile phase $100 \mathrm{mmol} / \mathrm{L}$ phosphate buffer ( $\mathrm{pH} 7.0$ ) containing $0.15 \mathrm{~mol} / \mathrm{L} \mathrm{NaCl}$ was pumped by the LC-10AD (Shimadzu, Kyoto, Japan) device at a flow rate of $0.5 \mathrm{~mL} / \mathrm{min}$. The samples of the volume of $20 \mu \mathrm{L}$ were injected by a 7725i-type Rheodyne valve. The SEC analysis performance was monitored on-line by a differential refractive index detector (RID-10, Shimadzu, Kyoto, Japan).

\subsection{SEC-MALS Analyses}

The molar mass distribution (MMD) of samples was determined by a modular multi-detector SEC system. This system consisted of an Alliance 2695 separation module from Waters (Milford, MA, USA) equipped with two on-line detectors: A multi-angle light scattering photometer (MALS Dawn DSP-F) from Wyatt (Santa Barbara, CA, USA) and a 2414 differential refractometer (DRI) from Waters (Milford, MA, USA); the latter was used as a polymer concentration detector. The setup of this multi-detector SEC system was serial in the following order: Alliance-MALS-DRI. The wavelength of the MALS laser was $632.8 \mathrm{~nm}$. The light scattering signal was detected simultaneously at fifteen scattering angles ranging from $14.5^{\circ}$ to $151.3^{\circ}$. The calibration constant was calculated using toluene as standard, assuming a Rayleigh factor of $1.406 \times 10^{-5} \mathrm{~cm}^{-1}$. The angular normalization was performed by measuring the scattering intensity of a concentrated solution of BSA (bovine serum albumin) globular protein in the mobile phase, assumed to act as an isotropic scatterer. Considering the high-molar-mass of the samples, a relatively low flow rate, $0.5 \mathrm{~mL} / \mathrm{min}$, was used to avoid shear-degradation of the polymer in the SEC columns. The experimental methodology for a reliable use of the SEC-MALS chromatographic system was described in detail [18-21]. The experimental conditions of the SEC-MALS system were the following: two Shodex (KB806 and KB805) columns from Tosoh Bioscience (Stuttgart, Germany); mobile phase: $0.20 \mathrm{~mol} / \mathrm{L}$ aqueous $\mathrm{NaCl}$; temperature: $35^{\circ} \mathrm{C}$; injection volume: $150 \mu \mathrm{L}$; polymer concentration: $0.4-1.0 \mathrm{mg} / \mathrm{mL}$ depending on the molar mass of the polymer sample. The refractive index increment, $\mathrm{dn} / \mathrm{dc}$, with respect to the $0.20 \mathrm{~mol} / \mathrm{L}$ aqueous $\mathrm{NaCl}$ solvent was measured by a KMX-16 differential refractometer from LDC Milton Roy (Riviera Beach, FL, USA). The $\mathrm{dn} / \mathrm{dc}$ value determined was $0.150 \mathrm{~mL} / \mathrm{g}$. 


\subsection{Non-Isothermal Chemiluminometry}

Chemiluminescence measurements were performed with a photon-counting instrument Lumipol 3, manufactured at the Polymer Institute of the Slovak Academy of Sciences (Bratislava, Slovakia). The sample was placed on an aluminum pan in the sample compartment. The gas flow (pure nitrogen or oxygen) through the sample cell was $3.0 \mathrm{~L} / \mathrm{h}$. The temperature in the sample compartment of the apparatus was raised from 40 up to $250{ }^{\circ} \mathrm{C}$, with a linear gradient of $2.5^{\circ} \mathrm{C} / \mathrm{min}$. The signal from the photocathode was recorded at 10 -s data collection intervals. The amount of samples used for each measurement was in the range of $1.5-3.2 \mathrm{mg}$.

\subsection{Non-Isothermal Thermogravimetry}

TGA - the thermogravimetric analyses were performed using a Mettler Toledo TGA/SDTA $851^{\mathrm{e}}$ instrument in a nitrogen or oxygen flow $(30 \mathrm{~mL} / \mathrm{min})$ using a heating rate of $10{ }^{\circ} \mathrm{C} / \mathrm{min}$ in a temperature range from room temperature up to $550^{\circ} \mathrm{C}$. Indium and aluminum were used for temperature calibration. The amount of samples applied ranged between 0.28 and $3.45 \mathrm{mg}$. Three parallel runs were performed for each sample.

\subsection{Differential Scanning Calorimetry}

Calorimetric measurements were performed using a Mettler Toledo DSC $821^{\mathrm{e}}$ apparatus. Indium was used for calibration of temperature and heat of fusion. Thermal stability of the samples was evaluated from the second heating of samples from room temperature up to $500{ }^{\circ} \mathrm{C}\left(10{ }^{\circ} \mathrm{C} / \mathrm{min}\right)$ in a nitrogen atmosphere $(50 \mathrm{~mL} / \mathrm{min})$. The first heating (from room temperature up to $170{ }^{\circ} \mathrm{C}$ ) was used for water removal. Thermooxidative decomposition was investigated in a temperature range from room temperature up to $500{ }^{\circ} \mathrm{C}\left(10{ }^{\circ} \mathrm{C} / \mathrm{min}\right)$ in oxygen flow $(50 \mathrm{~mL} / \mathrm{min})$. The amount of samples applied ranged between 0.80 and $2.77 \mathrm{mg}$. Three parallel runs were performed for each sample.

\section{Results and Discussion}

\subsection{Rotational Viscometry}

Degradation of high-molar-mass HA in the presence of a trace amount of $\mathrm{Cu}(\mathrm{II})$ ions $(1 \mu \mathrm{mol} / \mathrm{L})$ and ascorbic acid $(100 \mu \mathrm{mol} / \mathrm{L})$ as the reducing agent mimics the situation that may occur during the early stage of acute phase inflammative degradation of SF within a particular joint due to hydroxyl radicals. Chemistry of the well-established Weissberger's biogenic oxidative system (WBOS, Scheme 1) under aerobic conditions can be illustratively described as follows: 
Scheme 1. Chemistry of WBOS (Weisberger biogenic oxidative system). $\mathrm{AscH}^{-}$and DHA (dehydroascorbate) denote ascorbate anion and dehydroascorbate, respectively.
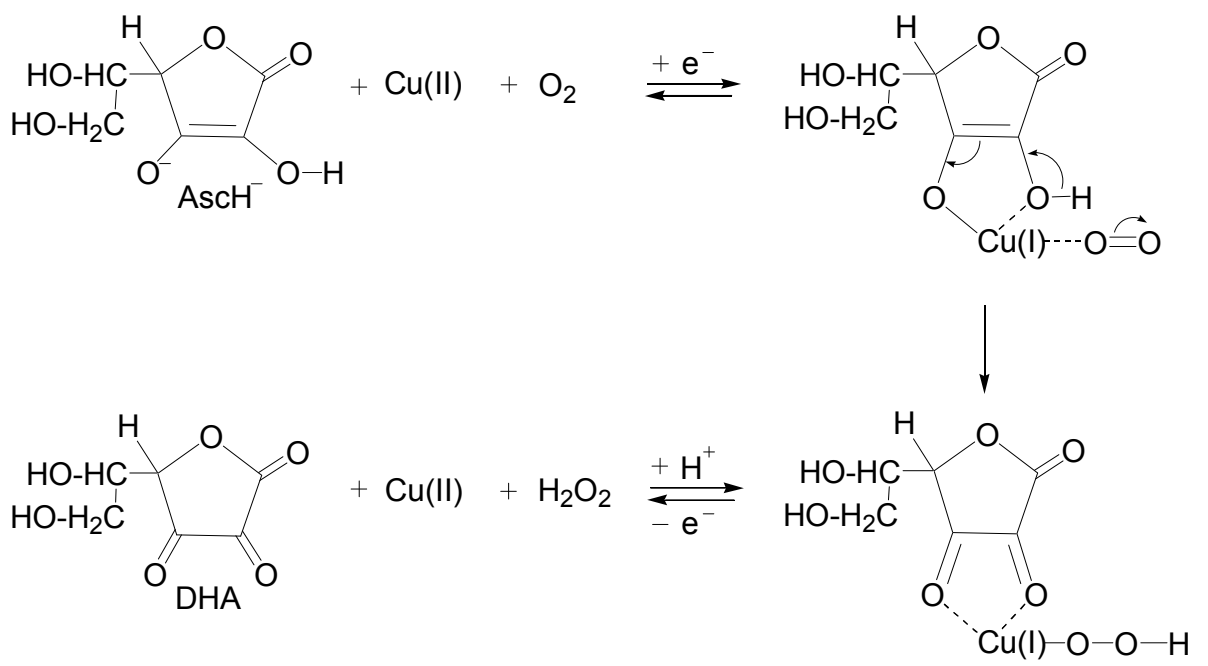

As evident, the molecules of oxygen by a sequence of cyclic reactions is converted to $\mathrm{H}_{2} \mathrm{O}_{2}$ molecules, which from an intermediate $\mathrm{Cu}(\mathrm{I})$-complex decompose fast to hydroxyl radicals according to the pseudo-Fenton reaction: $\mathrm{H}_{2} \mathrm{O}_{2}+\mathrm{Cu}(\mathrm{I}) \rightarrow \cdot \mathrm{OH}+\mathrm{HO}^{-}+\mathrm{Cu}(\mathrm{II}) \cdot \cdot \mathrm{OH}$ radicals can then initiate the free-radical degradative chain reaction [22-30] of HA. The independent investigation of the mechanism of WBOS action proved that all three reactants, i.e., ascorbic acid, $\mathrm{Cu}(\mathrm{II})$, and oxygen, were indispensable for initiation of the degradation of HA macromolecules. One should, however, bear in mind that it has been repeatedly established that most commercially available HAs contain a minute amount of transition metal ions including cupric ions [31-33].

Two different experimental arrangements have been used so far when the HA macromolecules undergo free-radical degradation by WBOS. First, the HA degradative process is uninhibited, second an inhibitor/scavenger can be introduced in the function to protect the biopolymer degradation. The latter procedure has been exploited broadly to characterize various antioxidants/drugs to act as preventive and/or chain-breaking species.

By adding the substance for testing its protective effect against oxidative degradation of HA in time $0 \mathrm{~min}$, i.e., at the onset of free-radical generation, the substance capability to scavenge $\cdot \mathrm{OH}$ radicals and, thus, to act as a preventive antioxidant was addressed.

By adding the substance $1 \mathrm{~h}$ after the reaction onset, we investigated the ability of the substance to scavenge alkoxyl- and/or alkylperoxyl-type radicals and, thus, to inhibit the process in its developed stage.

The interpretation of results may be complicated by the potential effect of thiol compounds on non-radical decomposition of intermediately formed hydrogen peroxide and by the possibility of the depletion of WBOS due to consumption of ascorbic acid in Fenton's like reaction cycle.

Figure 2 illustrates the decrease in dynamic viscosity $(\eta)$ of the HA solution. Degradation of hyaluronan was induced by $\mathrm{Cu}(\mathrm{II})$ plus ascorbic acid-Weissberger's biogenic oxidative system (curves coded i). The initial $\eta$ value decreased from 11.5 to $7.4 \mathrm{mPa} \cdot \mathrm{s}$ at the end of 5-h recording. Curve coded P0207-1A represents the $\eta$ values of native HA solution. Bucillamine $(100 \mu \mathrm{mol} / \mathrm{L})$, applied before initiating the oxidative degradation of HA, prevented any reduction of the solution dynamic viscosity value. Moreover, rheopexy of the solution was clearly registered (panel B, curve coded ii). 
As evident in Figure 2, panel B, curve coded iii, dynamic viscosity of the reaction mixture decreased from the initial value 11.5 to $11.1 \mathrm{mPa} \cdot \mathrm{s}$ during $1 \mathrm{~h}$. The addition of bucillamine in $100 \mu \mathrm{mol} / \mathrm{L}$ concentration led to only a slight decrease of $\eta$ to the value $10.7 \mathrm{mPa} \cdot \mathrm{s}$ in the course of the following $30 \mathrm{~min}$. Afterwards, in the time interval from $90 \mathrm{~min}$ to $5 \mathrm{~h}$, the dynamic viscosity of the reaction mixture altered minimally (Figure 2, panel B, curve coded iii).

Figure 2. Graphical record of dynamic viscosity $(\eta)$ values of: native HA biopolymer solution (P0207-1A), WBOS comprising reaction mixture (i) and bucillamine (100 $\mu \mathrm{mol} / \mathrm{L})$ action on oxidative HA degradation in vitro induced by WBOS in two experimental arrangements (ii, iii). The WBOS containing $1 \mu \mathrm{mol} / \mathrm{L} \mathrm{CuCl}_{2}$ plus $100 \mu \mathrm{mol} / \mathrm{L}$ ascorbic acid. The changes of dynamic viscosity values were recorded by rotational viscometer for $5 \mathrm{~h}$ and temperature of $25^{\circ} \mathrm{C}$. All solutions were prepared in $0.15 \mathrm{~mol} / \mathrm{L} \mathrm{NaCl}$. Panel (A), curve i represents the $\eta$ of WBOS and HA solution, curve P0207-1A represents the $\eta$ of native HA biopolymer coded P0207-1A; Panel (B), similarly, curve i represents the $\eta$ of WBOS and HA solution, curve ii represents the changes of $\eta$ in the presence of bucillamine $(100 \mu \mathrm{mol} / \mathrm{L})$ in the reaction mixture, when bucillamine was added at the beginning of the oxidative degradation of HA, curve iii represents the changes of $\eta$ in the presence of bucillamine $(100 \mu \mathrm{mol} / \mathrm{L})$ in the reaction mixture, when bucillamine was added after one hour of the oxidative HA degradation.
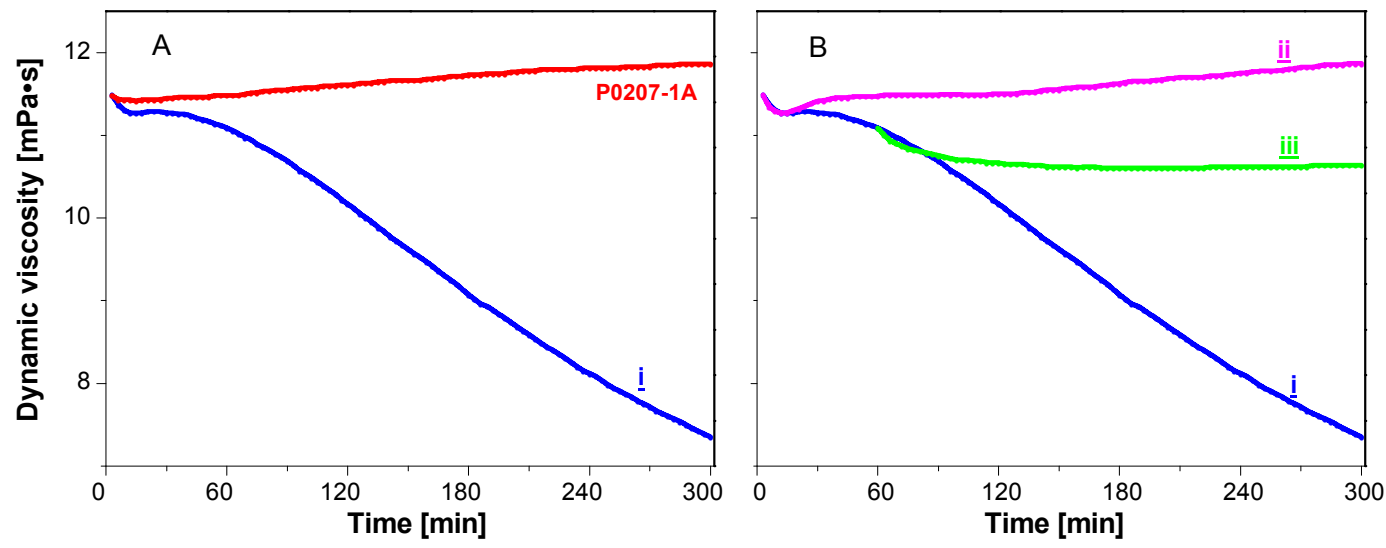

\subsection{SEC Analyses}

The primary analysis of the reaction mixtures carried out by a fast SEC method, considering especially the status at $5 \mathrm{~h}$ of the solution containing bucillamine and/or decomposed drug molecules (Figure 2, panel B, curve coded ii), indicated a shift of the chromatogram peak to a higher elution volume compared to the volume of native HA sample P0207-1A used in our study (not shown). The greater elution volume observed indicates lower hydrodynamic volume of the polymer solute-hence, the dissolved polymer is represented by lower molar mass. A further examination of the reaction mixture by a rapid SEC method confirmed our primary observation that although dynamic viscosity of the reaction mixture does not indicate alteration of the molecular parameters of the HA sample treated, the SEC elution properties of this solution did reveal some relevant changes. For this reason, our further investigation was focused on a detailed molecular characterization of the polymeric samples recovered from the reaction mixture in solid forms. 
Figure 3 displays the superimposition of the records of the 2414-DRI signal (the chromatograms) of four HA samples obtained by using the Alliance-MALS-DRI set. The elution volume window between approximately 12 and $21 \mathrm{~mL}$ represents the eluted polymeric fractions, while at the higher volume $(>21 \mathrm{~mL})$ the low-molar-mass species are eluted. A very good SEC separation is evident, especially for the native ultra-high-molar-mass (UHMM) HA sample. Records in Figure 3 demonstrate that the set of chromatographic columns used and the experimental conditions were adequate for an efficient SEC fractionation of all four HA samples.

Figure 3. Superimposition of DRI signal (analyte concentration) of four biopolymeric samples. The native HA sample P0207-1A, recovered polymers prepared by treating the native HA sample with WBOS without addition of bucillamine (i) and with addition of bucillamine in the system before the reaction onset (ii) and after $1 \mathrm{~h}$ (iii).

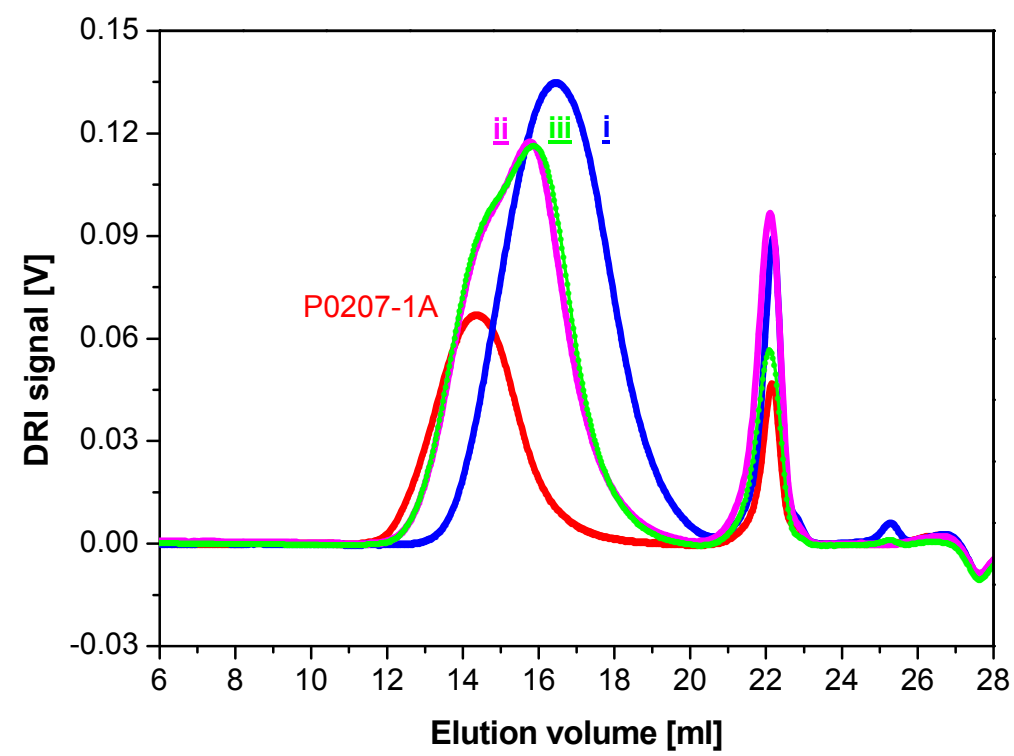

Figure 4 represents the comparison of differential MMD of all four samples. The molar mass of each polymeric fraction from the on-line MALS detector is absolute, not relative to some particular calibration standards, and the final molar mass distribution of the sample was obtained directly without any (relative) calibration. In experiments $\mathbf{i}$ without the presence of bucillamine, the significant reduction of the molar mass may be observed when compared with original intact HA. The both chromatograms and the MMD of ii and iii samples recovered at $5 \mathrm{~h}$ (Figures 3 and 4) show also the decrease of molar mass that is however, lower than that for experiment i. A small shoulder in the high-molar-mass fractions for experiments ii and iii may be observed as well. This is the most plausibly a consequence of the little extent of aggregation in these two samples, however, the aggregation appears to be marginal. The most important molecular characteristics related to SEC-MALS analyses are summarized in Table 1.

As is evident in Table 1, the native HA P0207-1A sample is an ultra-high-molar-mass sample (average molar mass $M_{\mathrm{w}}$ close to $\left.1 \mathrm{MDa}, R_{\mathrm{g}} \approx 105 \mathrm{~nm}\right)$ with relatively broad $\mathrm{MMD}\left(M_{\mathrm{w}} / M_{\mathrm{n}}=1.50\right)$. The slope $\alpha$ of the conformation plot of this sample (0.57) is typical of a native HA biopolymer. Several repeated experiments demonstrated high reproducibility of the P0207-1A degradation process by applying WBOS. For example, SEC-MALS measurements of samples ii resulted in the $M_{\mathrm{w}}$ values from 448.7 to $607.5 \mathrm{kDa}$. 
Figure 4. Comparison of differential MMD (molar mass distribution) of four biopolymeric samples. The native HA sample P0207-1A, recovered polymers prepared by treating the native HA sample with WBOS without addition of bucillamine (i) and with addition of bucillamine in the system before the reaction onset (ii) and after $1 \mathrm{~h}$ (iii).

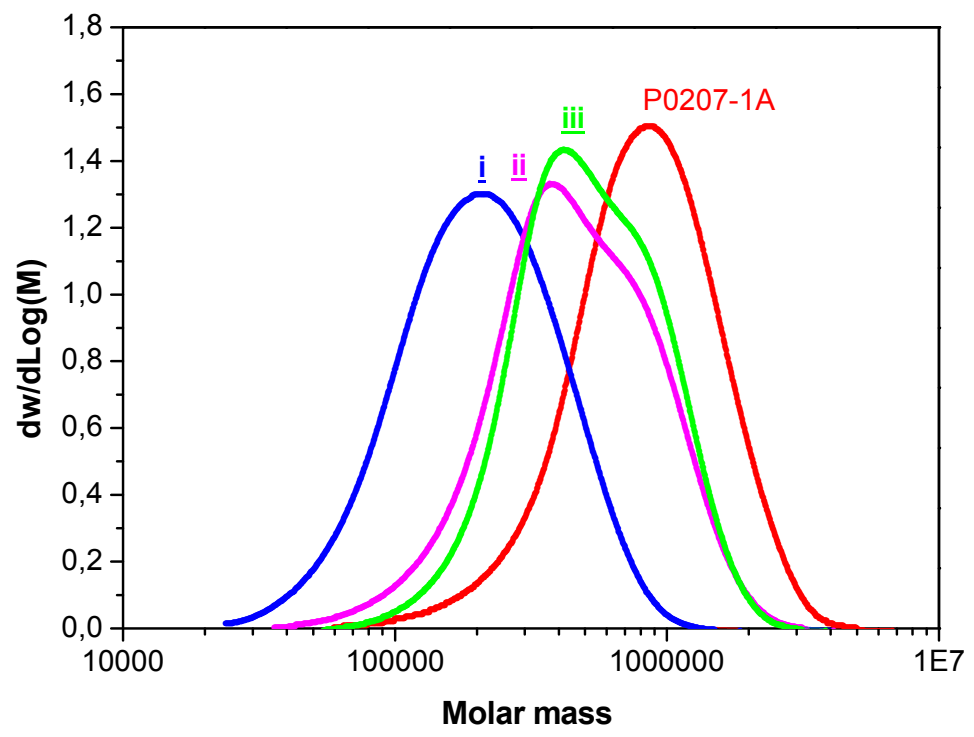

Table 1. Summary of SEC-MALS results of four samples.

\begin{tabular}{ccccccccc}
\hline Sample & $\begin{array}{c}\boldsymbol{M}_{\mathbf{p}} \\
(\mathrm{kDa})\end{array}$ & $\begin{array}{c}\boldsymbol{M}_{\mathbf{w}} \\
(\mathrm{kDa})\end{array}$ & $\begin{array}{c}\boldsymbol{M}_{\mathbf{w}} / \boldsymbol{M}_{\mathbf{n}} \\
(-/-)\end{array}$ & $\begin{array}{c}\boldsymbol{R}_{\mathbf{g}} \\
(\mathrm{nm})\end{array}$ & $\begin{array}{c}\boldsymbol{K} \\
(\mathbf{n m})\end{array}$ & $\boldsymbol{\alpha}$ & $\begin{array}{c}\text { Recovered Mass } \\
(\mathbf{\%})\end{array}$ & $\begin{array}{c}\boldsymbol{M}_{\mathbf{w}} \\
(\mathbf{d e c r e a s e}, \mathbf{\%})\end{array}$ \\
\hline P0207-1A & 886.1 & 970.4 & 1.50 & 105.1 & $4.27 \times 10^{-2}$ & 0.57 & 78.2 & 0.0 \\
i & 217.2 & 247.9 & 1.56 & 46.1 & $6.27 \times 10^{-2}$ & 0.53 & 79.9 & -74.5 \\
ii & 370.9 & 563.5 & 1.61 & 76.8 & $8.18 \times 10^{-2}$ & 0.52 & 83.2 & -41.9 \\
iii & 418.2 & 609.8 & 1.44 & 78.6 & $5.70 \times 10^{-2}$ & 0.54 & 84.5 & -37.2 \\
\hline
\end{tabular}

Comparison of the conformation plot $R_{\mathrm{g}}=f(\mathrm{M})$ of four samples is shown in Figure 5, panel A. The conformation plot in the form $R_{\mathrm{g}}=K \times M^{\alpha}$ links gyration radius $R_{\mathrm{g}}$ with chain length (molar mass) of macromolecules. In general, the conformation plot is very important because it supports the idea about possible polymer branching and potential derivatization or chemical modification of the polymer. Here the change/reduction of the molar mass with the extent of degradation is accompanied by the decrease of the gyration radius. The conformation plot of four samples clearly shows a molar mass decrease (to different extent) in the inspected/recovered $\mathbf{i}$, ii, and iii samples. The values of the slope $\alpha$ for these three samples (Table 1) were somewhat lower with respect to the native P0207-1A sample. This conformational result is most plausibly a consequence of the little extent of aggregation of these three samples, which, according to the above-mentioned facts, could be classified as hyaluronans or true HA fragments. 
Figure 5. (A) represents the dependences of gyration radius $\left(R_{\mathrm{g}}\right)$ on molar mass $(M)$. (B) illustrates $M v s$. the third power of gyration radius $\left(R_{\mathrm{g}}{ }^{3}\right)$, where the $R_{\mathrm{g}}$ values were taken at the end of curves of panel A. Native HA sample P0207-1A, recovered polymers prepared by treating native HA sample with WBOS without addition of bucillamine (i) and with addition of bucillamine in the system before the reaction onset (ii) and after $1 \mathrm{~h}$ (iii).
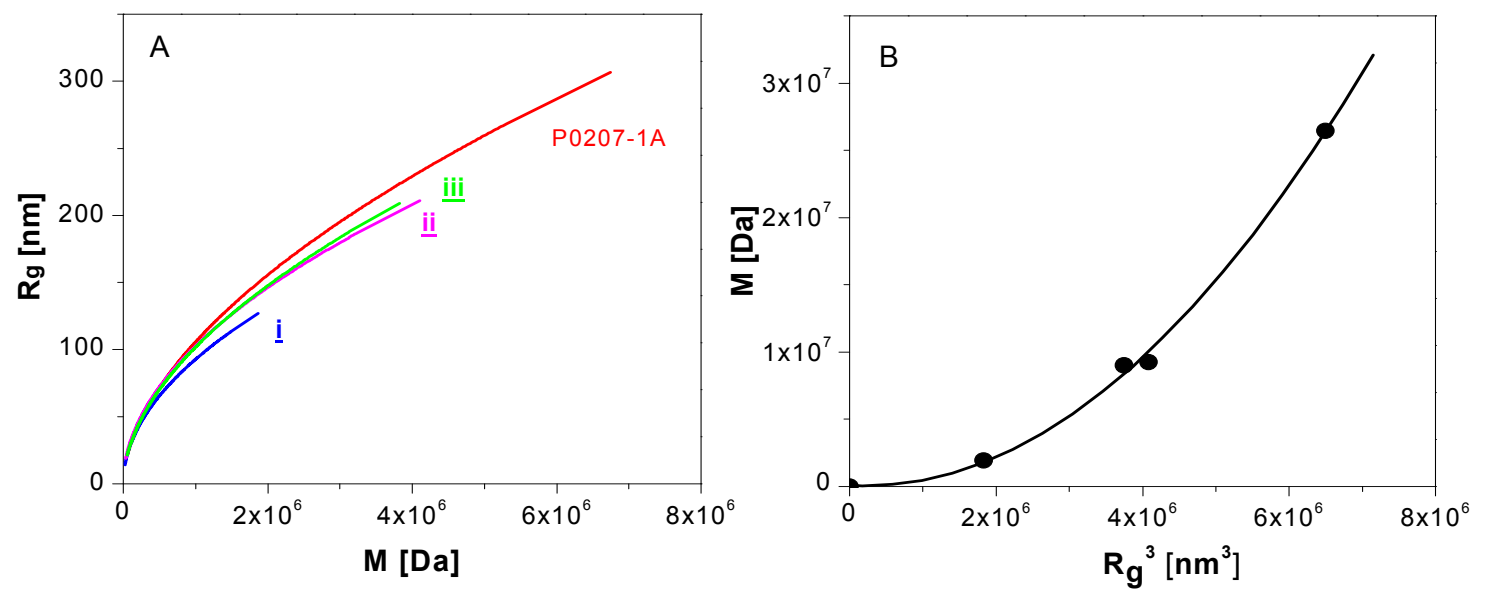

\subsection{Non-Isothermal Chemiluminometric, TGA, and DSC Characterization of the Four}

Biopolymeric Samples

The non-isothermal chemiluminometric, TGA, and DSC methods served for the investigation of four biopolymeric samples (P0207-1A, i, ii, and iii) enabling to evaluate the momentaneous state of fragmented hyaluronans towards (a) presence of hydroperoxides (inert atmosphere), (b) remaining oxidisability determined by the concentration of potential oxidation sites, (c) eventual tendency of fragmented samples to further transformation reactions such as crosslinking, etc. One has to remind again that reprecipitated samples were examined apparently lacking all low molar mass compounds and that records obtained were typical for the temperature and oxygen induced reactions of functional groups that appeared in HA after the reaction with WBOS. It is to be noted that in inert atmosphere (Figure 6) the chemiluminescence signal reflects the three mutually superimposed processes. At the lowest temperature it is the annealing of defect structures and structural abnormalities, at medium temperatures it is the bimolecular decomposition of hydroperoxides, and at the highest temperatures it is the scission of bonds linking the macromolecular chains. The structural defects and hydroperoxides are converted to radicals, of which recombination provides the heat necessary for the excitation of potential emitters, while their conversion into the ground state leads to light emission. On the other hand, in oxygen atmosphere we follow the oxidation of the fragmented sample as a whole. Secondary alkylperoxyl radicals, which are formed from bimolecular decomposition of hydroperoxides, are particularly "efficient" in such a process. They provide triplet carbonyls and singlet oxygen in a step which takes place in parallel (Scheme 2). The exothermicity of disproportionation reaction of secondary alkylperoxyl radicals is very high (around $420 \mathrm{~kJ} / \mathrm{mol}$ and higher). The recombination of alkoxyl-type radicals provides also some exothermicity $(320-400 \mathrm{~kJ} / \mathrm{mol})$, however the absence of emitters reduces the light intensity to a considerably lower value. That is why the chemiluminescence from hyaluronan in nitrogen where peroxyl radicals are provided only from the bimolecular decomposition of hydroperoxides is significantly lower (compare Figures 6 and 7). 
Figure 6. Non-isothermal chemiluminometry of four biopolymeric samples. The sample treatment was carried out in nitrogen. Native HA sample P0207-1A, recovered polymers prepared by treating native HA sample with WBOS without addition of bucillamine (i) and with addition of bucillamine in the system before the reaction onset (ii) and after $1 \mathrm{~h}$ (iii). The inserted plot (a) is a cross-section of the original runs where intensity of chemiluminescence is plotted to $10 \%$ of its value at $250{ }^{\circ} \mathrm{C}$.

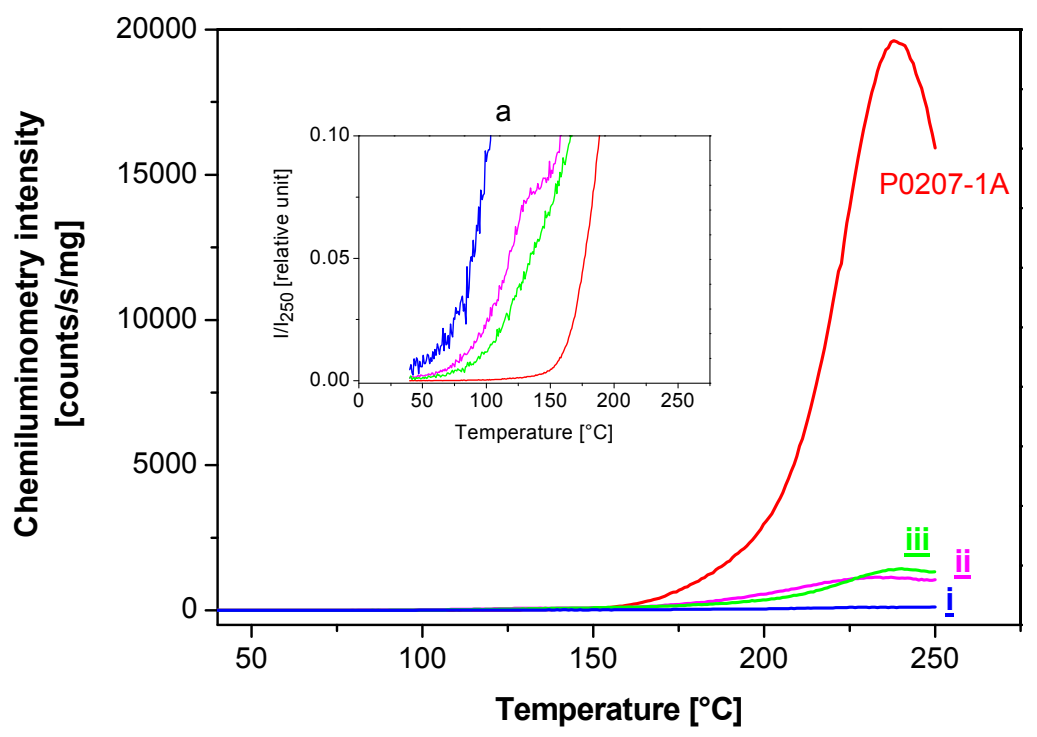

Scheme 2. Mechanism of the population of excited states from carbonyls and oxygen subsequently emitting light. Asterisks denote the excited states of ketones (triplet) and oxygen (singlet), which are converted to the ground state with the emission of light.

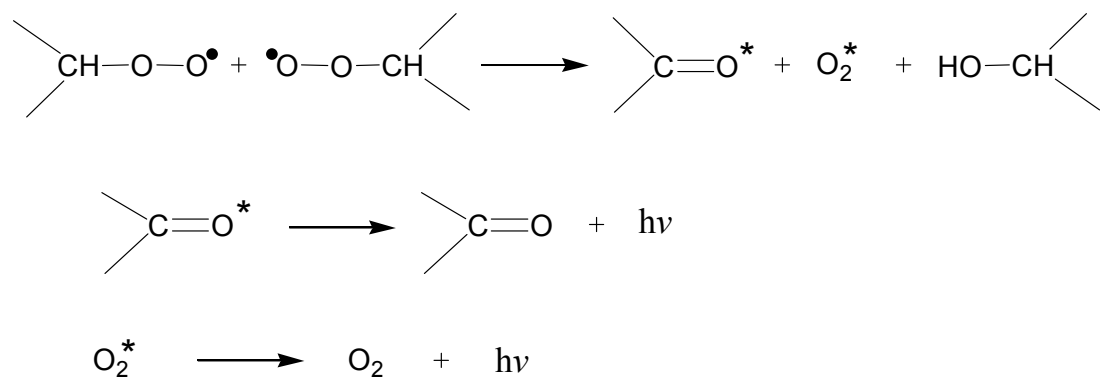

The results of non-isothermal chemiluminescence of four biopolymer samples, illustrated in Figures 6 and 7 , give the maximum of the light emission at temperatures above $200{ }^{\circ} \mathrm{C}$. The temperature of maximum chemiluminescence intensity in nitrogen is lower than that in oxygen. The inserted graph A underlines the luminescence behavior of the material in the region of lower temperatures. Figure 6 (red curve) illustrates that the native HA contains a certain amount of hydroperoxides as it performs a well-developed maximum in nitrogen situated at $238^{\circ} \mathrm{C}$. The non-isothermal chemiluminometric assay under oxygen atmosphere (Figure 7, red curve) revealed that the native HA was readily oxidized giving rise to a chemiluminescence about 15 times more intense than that in nitrogen (Figure 6, red curve). The assumed route is demonstrated in Scheme 3. The peroxidic compound which comes into consideration is likely to be peracid, formed from the oxidation of C6 carbon of the glucopyranosyl unit. This peracid is ultimately converted to acid. The another kind of peroxides may be formed after the free 
radical attack of $\mathrm{C} 3$ carbon atom of glucosamine or $\mathrm{C} 1$ atom of glucuronyl acid unit that are linked via the oxygen bridge. This occurs as the main chain scission.

Figure 7. Non-isothermal chemiluminometry of four biopolymeric samples. The samples were measured in oxygen. Native HA sample P0207-1A, recovered polymers prepared by treating native HA sample with WBOS without addition of bucillamine (i) and with addition of bucillamine in the system before the reaction onset (ii) and after $1 \mathrm{~h}$ (iii). The inserted plot (a) is a cross-section of the original runs in relative units where intensity of chemiluminescence is plotted to $10 \%$ of its maximum value.

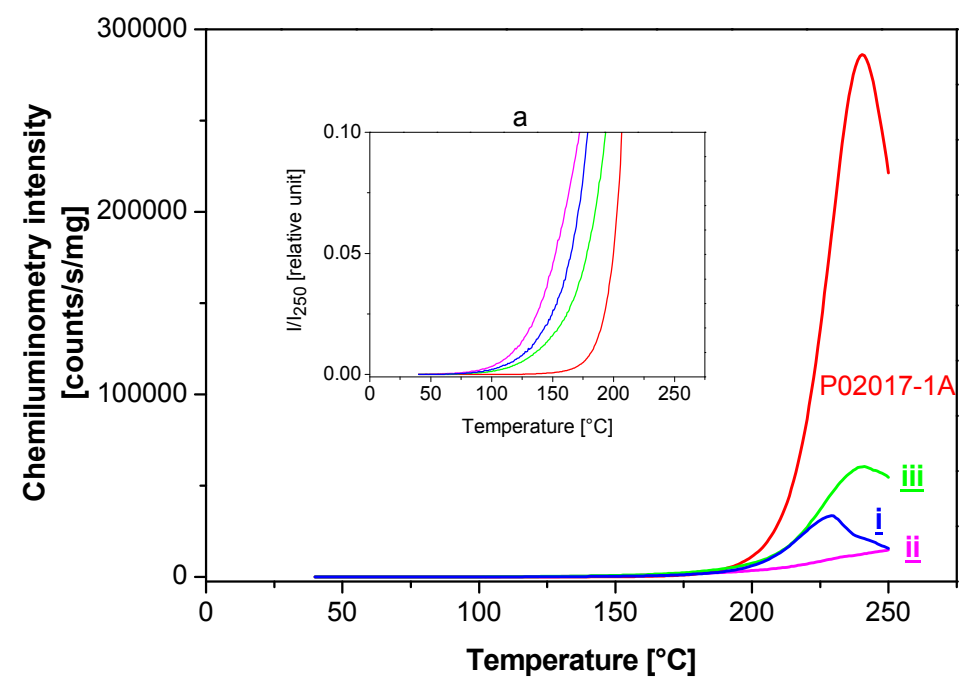

Scheme 3. The potential sites of the free-radical attack on HA.

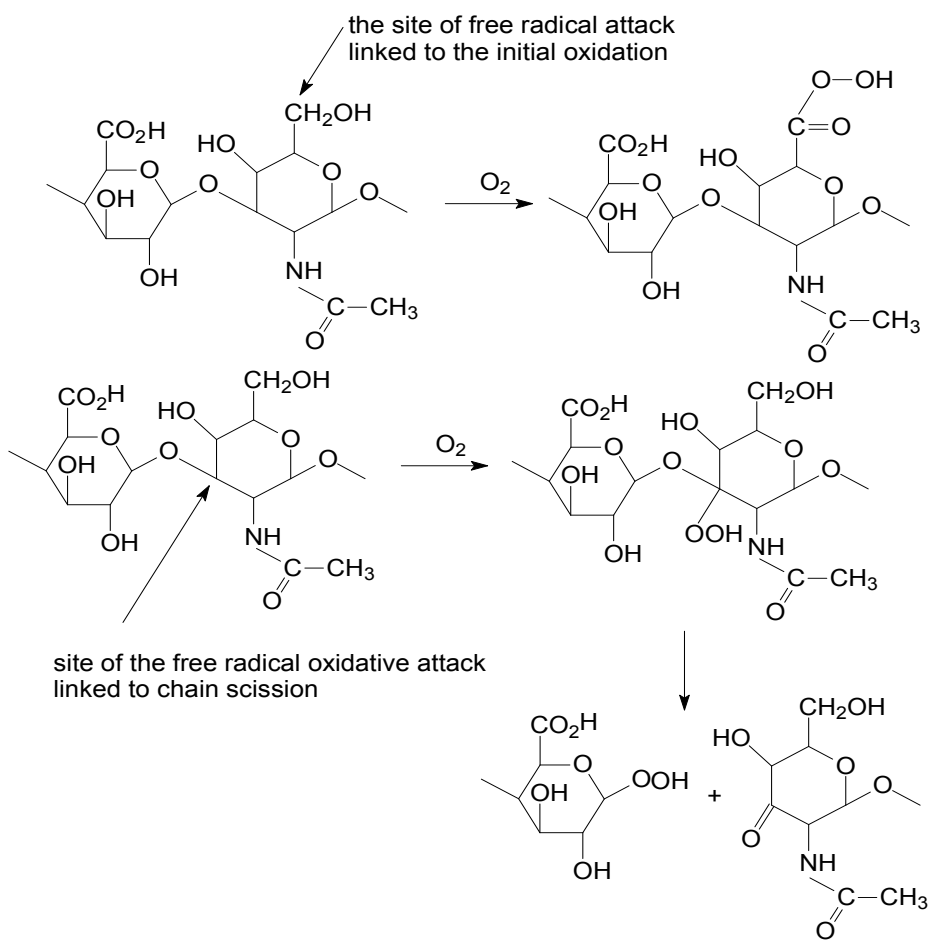

The non-isothermal chemiluminescence of samples i, ii, and iii (Figures 6 and 7) indicates a reduction of the signal intensity for fragmented samples to a rather low value. This is a common feature of all 
fragmented samples and is in accordance with the performance of $\mathrm{N}$-acetyl-L-cysteine reported in [15]. In Figures 6 and 7 we may also see the graphical metamorphosis of the plots of nonisothermal chemiluminescence for four biopolymer samples (inserts in Figures 6 and 7). The chemiluminescence intensity was expressed there in relative units as $I / I_{250}$, where $I_{250}$ is the light intensity at the last temperature reading $\left(250^{\circ} \mathrm{C}\right)$ and plotted until 10 p.c. of the whole graph. We see that samples ii and iii are, at low temperatures, less stable than the intact hyaluronan but more stable than that of sample $\mathbf{i}$. It is of interest that the molar mass $M_{\mathrm{w}}$ is also reduced, depending on the method of HA treatment (Figure 8). The decrease of the molar mass and the shift of the initial increase of chemiluminescence intensity to lower temperatures in nitrogen atmosphere correspond each to other. The initial increase of the chemiluminescence intensity in oxygen discriminates the fragmented samples in the sequence according to the facility of oxidation $\mathbf{i i}>\mathbf{i}>\mathbf{i i i}>$ P0207-1A. The chemiluminescence intensity Worth of further discussion is the finding that the sample denoted as ii did not change the viscosity in the rotational viscometer while its molar mass was reduced almost to one half of the original value (Table 1).

Figure 8. The plot of temperature of achieving $10 \%$ of the maximum chemiluminescence intensity expressed in relative units $v s$. molar mass $M_{\mathrm{w}}$. The sample treatments were carried out in oxygen $(\circ)$ and nitrogen $(\bullet)$.

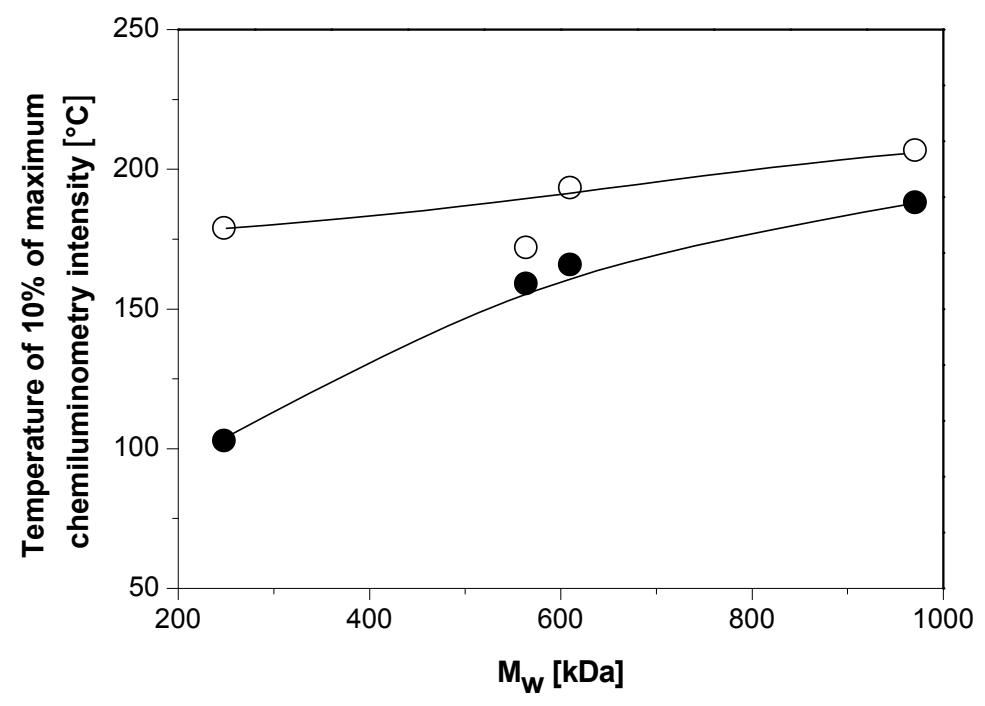

DSC records of both intact and fragmented hyaluronans appear to be rather complex involving endothermal release of low molar compounds absorbed (water) at low temperature not shown here and surprisingly also exotherms at increased temperatures. The temperature interval between 150 and $375^{\circ} \mathrm{C}$ seems to be the most representative for the characterization of HA. In nitrogen (Figure 9, panel A), the exotherms $\mathrm{M}$ and $\mathrm{N}$ may be seen. With the time of treatment in the rotational viscometer, the exotherms shift to lower temperatures. The most degraded sample $\mathbf{i}$ loses the exotherm $\mathrm{N}$ that was originally situated at temperatures around $270{ }^{\circ} \mathrm{C}$ and is replaced by the shoulder. The exotherm $\mathrm{M}$ is splitted into two parts. The disappearance of the exotherm $\mathrm{N}$ may be linked to the reduction of the molar mass. The same result was observed in the paper of Hrabárová et al. [15], where protective effects of $\mathrm{N}$-acetyl-L-cysteine against HA degradation were examined. DSC signals in oxygen (Figure 9, panel B) are much more pronounced compared to nitrogen (10-20 times), which is probably related to oxidation of HA molecules. Here, we again distinguish two exotherms $\mathrm{X}$ and $\mathrm{Y}$. Their maxima also shift to lower 
temperatures with the extent of HA degradation (Table 2). It is of interest to plot the surface below the DSC exotherms from Figure $9 v s$. the number of average molar mass of degraded samples (Figure 10). In the case of DSC nitrogen experiments the latter increases, the highest being for sample $\mathbf{i}$ while for DSC in oxygen the highest being for intact sample. This decrease corresponds to the decrease of maximum chemiluminescence intensity with the extent of HA degradation in oxygen experiments (Figure 7) and also to the depletion of oxidizable sites in HA. It is surprising that DSC records in nitrogen where mainly the degradation is expected give exotherms. Thus, it may be deduced that fragmented samples contain two kinds of sites:

a Oxidizable sites that are revealed in oxygen atmosphere,

b The sites capable of crosslinking or back polymerization that are exothermic that are revealed in a nitrogen atmosphere.

Figure 9. DSC records after the subtraction of the slope line (within the temperature interval 150 and $300^{\circ} \mathrm{C}$ for nitrogen (A) and 150 and $375^{\circ} \mathrm{C}$ for oxygen (B)) of four biopolymeric samples. Native HA sample P0207-1A, recovered polymers prepared by treating native HA sample with WBOS without addition of bucillamine (i) and with addition of bucillamine in the system before the reaction onset (ii) and after $1 \mathrm{~h}$ (iii).
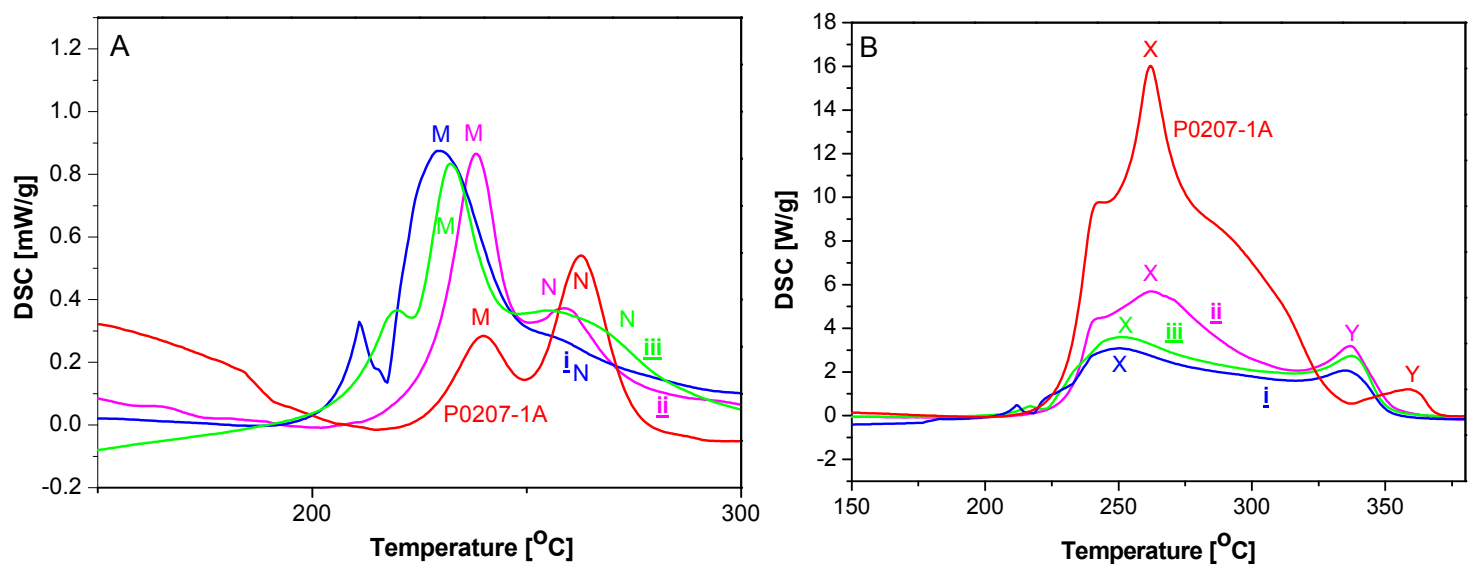

Table 2. Temperatures of the maximum DSC exotherms and residual amount of the sample after the thermogravimetry experiment at $350{ }^{\circ} \mathrm{C}$.

\begin{tabular}{cccccccc}
\hline \multirow{2}{*}{ Sample } & \multicolumn{4}{c}{ Temperature of exotherm, ${ }^{\circ} \mathbf{C}$} & & \multicolumn{2}{c}{ Amount of residue at $\mathbf{3 5 0}^{\circ} \mathbf{C}, \boldsymbol{\%}$} \\
\cline { 2 - 4 } & M & N & X & Y & & Nitrogen & Oxygen \\
\hline P0207-1A & 239 & 262 & 262 & 359 & & 45.6 & 33.6 \\
ii & 238 & 258 & 262 & 337 & & 44.4 & 43.2 \\
iii & 218,232 doublet & 250 & 250 & 337 & & 52.4 & 50.9 \\
i & 211,229 doublet & - & 248 & 334 & & 54.8 & 49.4 \\
\hline
\end{tabular}


Figure 10. The surface below the DSC exotherms for oxygen (squares) and nitrogen (circles) experiments plotted $v s$. molar mass of respective samples.

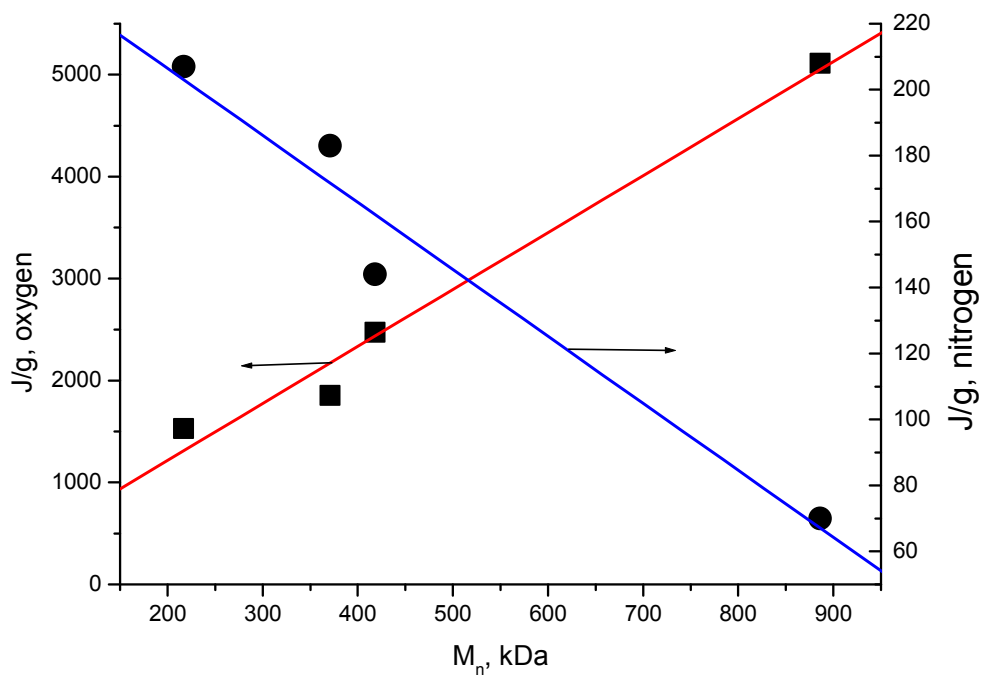

Nonisothermal thermogravimetry records also show that more fragmented samples of HA are less stable than intact ones. The curves for less stable samples are shifted to lower temperatures (Figure 11). The amount of residue that remains on the thermogravimetry pan is significantly higher when compared with intact sample, which may be tentatively ascribed to the additional reactions taking place in fragmented samples when the temperature increases. The higher residue should then correspond with larger extent of additional crosslinking or back polymerization.

Figure 11. TG (thermogravimetry) records of four biopolymeric samples. The sample treatment was carried out in nitrogen (A) and oxygen (B). Native HA sample P0207-1A, recovered polymers prepared by treating native HA sample with WBOS without addition of bucillamine (i) and with addition of bucillamine in the system before the reaction onset (ii) and after $1 \mathrm{~h}$ (iii).
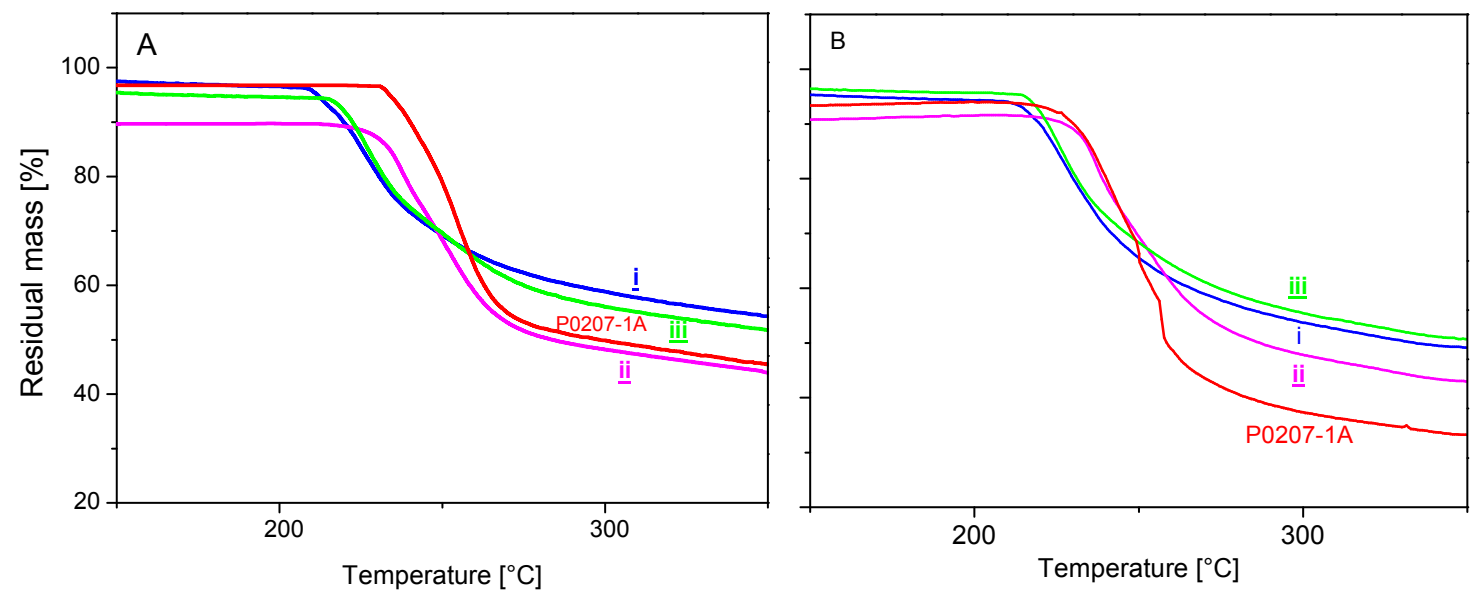

Hyaluronan is an outstanding biopolymer among glycosaminoglycans (GAGs), HA, chondroitin sulfate, heparan sulfate, dermatan sulfate, and keratan sulfate. Contrary to these four sulphates, HAs are represented by extremely high molar masses-HA macromolecules are extruded extracellularly by type B fibroblasts. One of the characteristic differences between HA and other GAGs is that HA contains no 
sulphur atom. Due to the high molar mass of native HA, viscoelasticity of its aqueous solutions is high. However during inflammations (e.g., rheumatoid arthritis), HA degradation was unequivocally claimed [34,35]. Thus, viscoelasticity of SF can decrease significantly. The phenomenon of an extensive decrease of dynamic viscosity of the aqueous solutions of degraded HAs has been exploited in studies of free-radical scavenging properties of various antioxidants [36,37]. Several investigators demonstrated that thiol type compounds ( $\mathrm{R}-\mathrm{SH})$, either endogenous or exogenous, can affect both initiation and propagation stages of HA free-radical degradation. The energy of the $\mathrm{S}-\mathrm{H}$ bond equals $\approx 30 \mathrm{kcal} / \mathrm{mol}$ and the compounds may efficiently act as scavengers of $\bullet \mathrm{OH}, \mathrm{ROO} \bullet$ and other free radicals. Several DMARDs can be ranked among thiol compounds (D-penicillamine, aurothiomalate, etc.). One molecule of bucillamine, a dithiol compound, is a source of either one or even two $\mathrm{H}$ atoms capable of reacting with free radicals. The observation of positive therapeutic action of bucillamine under RA conditions calls for in vitro studies modeling the situation which may occur in an inflamed joint. The results represented in Figure 2, curve i, illustrate changes of dynamic viscosity of the HA solution whose degradation was initiated by $\bullet \mathrm{OH}$ radicals. Under aerobic conditions, ascorbate is converted to $\mathrm{H}_{2} \mathrm{O}_{2}$, which in the presence of cuprous ions can generate reactive hydroxyl radicals $\bullet \mathrm{OH}$. It should be pointed out that higher ascorbate concentrations and/or $\mathrm{Cu}$ (II) ions can pronounce HA degradation and vice versa $[38,39]$. The fate of HA in the reaction mixture was monitored by simple SEC analyses and an extensive HA macromolecule degradation was observed. In the 5th hour the decrease of the $\eta$ value represented $\approx 64.3 \%$ (Figure 2, curve i). The curves ii and iii illustrated in Figure 2, panels A and B, could lead to a premature conclusion that bucillamine can totally scavenge all free-radicals within the reaction mixture. Simple SEC analyses indicated a relevant accelerating molar mass reduction and analyses performed in the 5 th hour by using $20 \mu \mathrm{L}$ probes proved HA degradation. Since a direct injection of the radical reaction mixture cannot be carried out in a SEC-MALS apparatus, an inevitable step was to terminate the degradative process and separate the polymer. The three samples $\mathbf{i}$, ii and iii in the solid form underwent a supplementary FT-IR (Fourier transform infrared spectroscopy) and inductively coupled plasma mass spectrometric analyses (ICP-MS). As proved, neither sample ii nor sample iii contained sulfur atom and the FT-IR records of all four samples (P0207-1A, i, ii and iii) were qualitatively identical (not shown). As shown in Table 1, the recovered samples ii and iii are molecularly different from the native P0207-1A. Minor differences of $R_{\mathrm{g}} v s . M$ plots (Figure 5, panel A) may be an indication of a different content of hydroperoxides in these four samples, as clearly evidenced in Figures 6 and 7. Since SEC-MALS analyses demonstrated no branching and no extensive modification of these four polymer samples, the native HA, as well as the samples i, ii and iii, can be classified as homopolymers. A prominent finding is the fact that the action of ascorbate plus $\mathrm{Cu}$ (II) in the absence and presence of bucillamine that reduces the chemiluminescence intensity performed with reprecipitated samples from rotational viscometer. Apparently the level of hydroperoxides that are formed in samples having removed all low molar mass additives is markedly reduced (Figure 6). The observation that the native HA P0207-1A contained a relevant amount of hydroperoxides, while samples i, ii and iii were practically hydroperoxide-free may partly explain differences in DSC records observed in these four samples. Contrary to sample P0207-1A, the higher and different residue content after thermogravimetry measurements of recovered polymers prepared by treating native HA sample with WBOS without and with addition of bucillamine in the system requires additional studies. If $\mathrm{Na}_{2} \mathrm{O}$ and $\mathrm{NaNO}_{3}$ are considered as the components of the residue from sodium hyaluronate, their residual mass should be around $7.5 \%$ 
and $20.9 \%$, respectively. Stability of the dynamic viscosity during HA radical degradation by ascorbate plus $\mathrm{Cu}(\mathrm{II})$ in the presence of bucillamine is indeed a very interesting observation (Figure 2, panels A and B). We have revealed that, similarly to other free radical oxidations of chain mechanism, the inhibitor, in our case bucillamine, may slow down free radical mediated degradation of the substrate but it did not stop it. Regardless of it HA degraded in the samples ii and iii, kept the $\eta$ value recorded by rotational viscometry (Figure 2, panel B, curve iii) or increased even slightly (curve ii) for at least five hours. In the literature [40], we may find that bovine synovial fluid and albumin solutions of similar concentration are rheopectic, i.e., their viscosity increases with time in steady shear. This unusual flow characteristic is caused by protein aggregation, and the total stress is enhanced by entanglement of this tenuous protein network with the long-chain polysaccharide sodium hyaluronate under physiological conditions. Neutron scattering measurements on albumin solutions demonstrate protein aggregation and all measurements are consistent with a weak dipolar attraction energy (of order 3kT) that is most likely augmented by hydrophobic interactions and/or disulfide bond formation between proteins. Our case may have something in common with it. On the one hand HA is fragmented and partially oxidized and its molar mass decreases. On the other hand, a sulphur compound may enhance the dragging of lower molar mass fragments into the coils of hyaluronans by association with oxidized structures and due to the shear stress the rheopexy may occur with parallel increase of viscosity. The similarity of the structural moieties in bucillamine with $\mathrm{N}$-acetyl groups of glucosamine units may contribute to it. These two opposite phenomena, namely the reduction of molar mass due to degradation and rheopexy due to association mediated by sulphur compounds induced by the shear stress in the rotational viscometer, may temporarily compensate each other.

\section{Conclusions}

Maximum chemiluminescence intensity corresponding to the momentaneous level of hydroperoxides in both oxygen and nitrogen atmosphere was significantly decreased by acting WBOS. This may be due to the essential reduction of the remaining potential sites of oxidation situated at $\mathrm{C} 6$ or at $\mathrm{C} 3$ carbon atoms of the glucosamine units. One should be, however, aware of the fact that chemiluminescence and thermal analysis tests reflect the momentaneous state of hyaluronan recovered after its degradation due to WBOS.

The important finding is that the viscosity of the HA solution in the rotational viscometer did not decrease by acting WBOS after adding $100 \mu \mathrm{M}$ bucillamine but the molar mass of HA in the solution was reduced, however in significantly lesser extent than those without the presence of bucillamine.

Similarly as in the case of $\mathrm{N}$-acetyl-L-cysteine, addition of bucillamine to fragmented HA leads to the significant changes of chemiluminescence intensity of the sample measured after its fragmentation.

This coincides with the disappearance of DSC exotherm at $270{ }^{\circ} \mathrm{C}$ and its replacement by the shoulder, as well as with the significant shift of all exotherms of fragmented HAs to lower temperatures, the fragmented HA being characterized under the same conditions as in chemiluminescence runs.

\section{Acknowledgments}

The authors thank Fumio Tsuji from Santen Pharmaceutical Co., Ltd. Osaka, Japan, for providing bucillamine. We are indebted to Julian Wills and Burkhard Stehl from ThermoFisher Scientific Inc., 
Vienna, Austria, for performing the ICP-MS measurements. The work was supported by the VEGA grants 2/0011/11, 2/0147/12, 2/0149/12, the Structural Funds of EU (ITMS 26240220040), and the APVV grant No.: 0351-10.

\section{Author Contributions}

Mária Baňasová performed the viscometric experiments, Jozef Rychlý performed the chemiluminometric experiments, Katarína Csomorová and Ivica Janigová performed the DSC and the TGA experiments, Danica Mislovičová performed the HPLC, Raniero Mendichi performed the SEC-MALS analyses. Mária Baňasová, Ladislav Šoltés, Ivo Juránek, Katarína Valachová and Jozef Rychlý wrote the paper.

\section{Conflicts of Interest}

The authors declare no conflict of interest.

\section{References}

1. Bishop, P.N. Structural macromolecules and supramolecular organisation of the vitreous gel. Prog. Retin. Eye Res. 2000, 19, 323-344.

2. Nickerson, C.S.; Kornfield, J.A. A “cleat” geometry for suppressing wall slip. J. Rheol. 2005, 49, 865-874.

3. Hrabárová, E.; Valachová, K.; Rychlý, J.; Rapta, P.; Sasinková, V.; Malíková, M.; Šoltés, L. High-molar-mass hyaluronan degradation by Weissberger's system: Pro- and anti-oxidative effects of some thiol compounds. Polym. Degrad. Stab. 2009, 94, 1867-1875.

4. Rychlý, J.; Šoltés, L.; Stankovská, M.; Janigová, I.; Csomorová, K.; Sasinková, V.; Kogan, G.; Gemeiner, P. Unexplored capabilities of chemiluminescence and thermoanalytical methods in characterization of intact and degraded hyaluronans. Polym. Degrad. Stab. 2006, 91, 3174-3184.

5. Collins, M.N.; Birkinshaw, C. Hyaluronic acid based scaffolds for tissue engineering-A review. Carbohydr. Polym. 2013, 92, 1262-1279.

6. Nitzan, D.W.; Nitzan, U.; Dan, P.; Yedgar, S. The role of hyaluronic acid in protecting surface-active phospholipids from lysis by exogenous phospholipasae A2. Rheumatology 2001, 40, 336-340.

7. Rinaudo, M.; Rozand Y.; Mathieu, P.; Conrozier, T. Role of different pre-treatments on composition and rheology of synovial fluids. Polymers 2009, 1, 16-34.

8. Furth, G.; Knierim, R.; Buss, V.; Mayer, C. Binding of bivalent cations by hyaluronate in aqueous solution. Int. J. Biol. Macromol. 2008, 42, 33-40.

9. Kogan, G.; Šoltés, L.; Stern, R.; Gemeiner, P. Hyaluronic acid: A natural biopolymer with a broad range of biomedical and industrial applications. Biotechnol. Lett. 2007, 29, 17-25.

10. Lepperdinger, G.; Fehrer, C.; Reitinger, S. Chemistry and Biology of Hyaluronan; Garg, H.G., Hales, C.A., Eds.; Elsevier: Boston, MA, USA, 2004; Volume 1, p.72.

11. Laurent, T.C.; Laurent, U.B.; Fraser, J.R. Serum hyaluronan as disease marker. Ann. Med. 1996, $28,241-253$. 
12. Fraser, J.R.; Laurent, T.C.; Laurent, U.B. Hyaluronan: Its nature, distribution, functions and turnover. J. Intern. Med. 1997, 242, 27-33.

13. Juránek, I.; Šoltés, L. Reactive oxygen species in joint physiology: Possible mechanism of maintaining hypoxia to protect chondrocytes from oxygen excess via synovial fluid hyaluronan peroxidation. In Kinetics, Catalysis and Mechanism of Chemical Reactions: From Pure to Applied Science, 1st ed.; Islamova, R.M., Kolesov, S.V., Zaikov, G.E., Eds.; Nova Science Publishers: New York, NY, USA, 2012; Volume 2, pp. 1-10.

14. Šoltés, L.; Mendichi, R.; Kogan, G.; Schiller, J.; Stankovská, M.; Arnhold, J. Degradative action of reactive oxygen species on hyaluronan. Biomacromolecules 2006, 7, 659-668.

15. Hrabárová, E.; Rychlý, J.; Sasinková, V.; Valachová, K.; Janigová, I.; Csomorová, K.; Juránek, I.; Šoltés, L. Structural characterisation of thiol-modified hyaluronans. Cellulose 2012, 19, 2093-2104.

16. Weissberger, A.; LuValle, J.E.; Thomas, D.S., Jr. Oxidation processes. XVI. The autoxidation of ascorbic acid. J. Am. Chem. Soc. 1943, 65, 1934-1939.

17. Khan, M.M.; Martell, A.E. Metal ion and metal chelate catalyzed oxidation of ascorbic acid by molecular oxygen. I. Cupric and ferric ion catalyzed oxidation. J. Am. Chem. Soc. 1967, 89, 4176-4185.

18. Mendichi, R.; Schieroni, A.G.; Grassi, C.; Re, A. Characterization of ultra-high molar mass hyaluronan: 1. Off-line static methods. Polymer 1998, 39, 6611-6620.

19. Mendichi, R.; Schieroni, A.G. Fractionation and characterization of ultra-high molar mass hyaluronan: 2. On-line size exclusion chromatography methods. Polymer 2002, 43, 6115-6121.

20. Wyatt, P.J. Light scattering and the absolute characterization of macromolecules. Anal. Chim. Acta 1993, 272, 1-40.

21. Mendichi, R.; Šoltés, L.; Schieroni, A.G. Evaluation of radius of gyration and intrinsic viscosity molar mass dependence and stiffness of hyaluronan. Biomacromolecules 2003, 4, 1805-1810.

22. Li, M.; Rosenfeld, L.; Vilar, R.E.; Cowman, M.K. Degradation of hyaluronan by peroxynitrite. Arch. Biochem. Biophys. 1997, 341, 245-250.

23. Praest, B.M.; Greiling, H.; Kock, R. Effects of oxygen-derived free radicals on the molecular weight and the polydispersity of hyaluronan solutions. Carbohydr. Res. 1997, 303, 153-157.

24. Al-Assaf, S.; Hawkins, C.L.; Parsons, B.J.; Davies, M.J.; Phillips, G.O. Identification of radicals from hyaluronan (hyaluronic acid) and cross-linked derivatives using electron paramagnetic resonance spectroscopy. Carbohydr. Polym. 1999, 38, 17-22.

25. Jahn, M.; Baynes, J.W.; Spiteller, G. The reaction of hyaluronic acid and its monomers, glucuronic acid and $N$-acetylglucosamine, with reactive oxygen species. Carbohydr. Res. 1999, 321, 228-234.

26. Rees, M.D.; Hawkins, C.L.; Davies, M.J. Polysaccharide fragmentation induced by hydroxyl radicals and hypochlorite. In Hyaluronan: Chemical, Biochemical and Biological Aspects; Kennedy, J.F., Phillips, G.O., Williams, P.A., Hascall, V.C., Eds; Woodhead Publishers: Cambridge, UK, 2002; Volume 1, p. 151.

27. Gao, F.; Koenitzer, J.R.; Tobolewski, J.M.; Jiang, D.; Liang, J.; Noble, P.W.; Oury, T.D. Extracellular superoxide dismutase inhibits inflammation by preventing oxidative fragmentation of hyaluronan. J. Biol. Chem. 2008, 283, 6058-6066. 
28. Kennett, E.C.; Davies, M.J. Glycosaminoglycans are fragmented by hydroxyl, carbonate, and nitrogen dioxide radicals in a site-selective manner: Implications for peroxynitrite-mediated damage at sites of inflammation. Free Radic. Biol. Med. 2009, 47, 389-400.

29. Yang, S.; Guo, Z.; Miao, F.; Xue, Q.; Qin, S. The hydroxyl radical scavenging activity of chitosan, hyaluronan, starch and their O-carboxymethylated derivatives. Carbohydr. Polym. 2010, 82, 1043-1045.

30. Darzynkiewicz, Z.; Balazs, E.A. Genome integrity, stem cells and hyaluronan. Aging 2012, 4, 78-88.

31. Swann, D.A. The degradation of hyaluronic acid by ascorbic acid. Biochem. J. 1967, 102, 42-44.

32. Hofmann, H.; Schmut, O. The inability of superoxide dismutase to inhibit the depolymerization of hyaluronic acid by ferrous ions and ascorbate. Albrecht. von Graefes. Arch. Klin. Ophthalmol. 1980, 214, 181-185.

33. Wong, S.; Halliwell, B.; Richmond, R.; Skowroneck, W.R. The role of superoxide and hydroxyl radicals in the degradation of hyaluronic acid induced by metal ions and by ascorbic acid. J. Inorg. Biochem. 1981, 14, 127-134.

34. Hajjaji, H.E.; Cole, A.A.; Manicourt, D.H. Chondrocytes, synoviocytes and dermal fibroblasts all express PH-20, a hyaluronidase active at neutral pH. Arthritis Res. Ther. 2005, 7, 756-768.

35. Hutadilok, N.; Ghosh, P.; Brooks, P.M. Binding of haptoglobin, inter-a-trypsin inhibitor, and a, proteinase inhibitor to synovial fluid hyaluronate and the influence of these proteins on its degradation by oxygen derived free radicals. Ann. Rheum. Dis. 1988, 47, 377-385.

36. Mendichi, R.; Audisio, G.; Facino, R.M.; Carini, M.; Schieroni, A.G.; Saibene, L. Use of size exclusion chromatography to study the protective effect of radical scavengers on oxygen free-radical-induced degradation of hyaluronic acid. Int. J. Polym. Anal. Charact. 1995, 1, 365-371.

37. Mendoza, G.; Alvarez, A.I.; Pulido, M.M.; Molina, A.J.; Merino, G.; Real, R.; Fernandes, P.; Prieto, J.G.; Inhibitory effects of different antioxidants on hyaluronan depolymerization. Carbohydr. Res. 2007, 342, 96-102.

38. Harris, M.J.; Herp, A.; Pigman, W. Metal catalysis in the depolymerization of hyaluronic acid by autoxidants. J. Am. Chem. Soc. 1972, 94, 7570-7572.

39. Liu, K.M.; Swann, D.; Lee, P.; Lam, K.W. Inhibition of oxidative degradation of hyaluronic acid by uric acid. Curr. Eye Res. 1984, 3, 1049-1053.

40. Oates, K.M.N.; Krause, W.E.; Jones, R.L.; Colby, R.H. Rheopexy of synovial fluid and protein aggregation. J. R. Soc. Interface 2006, 3, 167-174.

(C) 2014 by the authors; licensee MDPI, Basel, Switzerland. This article is an open access article distributed under the terms and conditions of the Creative Commons Attribution license (http://creativecommons.org/licenses/by/4.0/). 